\title{
Dimorfismo sexual por meio de medidas lineares da escápula
}

\author{
Sexual dimorphism through linear measurements of scapula
}

Maria Julia Assis Vicentim ${ }^{1}$

Viviane UlbRicht ${ }^{\mathrm{I}}$

CRisthiane Martins SCHMidT

Francisco Carlos Groppo ${ }^{\mathrm{I}}$

Eduardo Daruge JUnior ${ }^{\mathrm{I}}$

Sônia Maria de Stefano Piedade

Jỗo Cesar Barbieri BEDRAN DE

CASTRO

Carlos Alberto Sassi Etche-

GOYEN $^{1}$

LUIZ FRANCESQUINI JUNIOR ${ }^{1}$

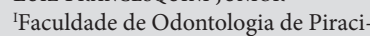

caba (Fop/Unicamp). Piracicaba/

SP - Brasil

"Escola Superior de Agricultura Luiz de Queiroz (ESALQ/USP). Piracicaba/SP - Brasil
Resumo A partir do paradigma de que a determinação do sexo post mortem pode ser realizada em qualquer osso humano, o presente estudo observacional analítico foi feito em 195 ossadas de brasileiros, sendo 109 do sexo masculino e 86 do sexo feminino, existentes no Biobanco Osteológico e Tomográfico Prof. Dr. Eduardo Daruge da FOP/UNICAMP, que não mostravam anomalias de crescimento e estão na faixa de idade de 18 a 100 anos. Teve como objetivo estudar sete medidas lineares na escápula direita (A - comprimento máximo da cavidade glenoidal, B largura máxima da cavidade glenoidal, $\mathrm{C}$ - ângulo superior da escápula - ângulo inferior, D - ângulo superior - ângulo lateral da escápula, $\mathrm{E}$ - ângulo lateral - ângulo inferior da escápula, F - borda mais equidistante do acrômio - ângulo superior da escápula e G - comprimento do acrômio) visando verificar se as mesmas são dimórficas e construir um modelo matemático para a determinação do sexo post mortem. Os resultados indicaram que todas as medidas estudadas são dimórficas e foi possível estabelecer um modelo matemático com quatro medidas (A - comprimento máximo da cavidade glenoidal, B - largura máxima da cavidade glenoidal, C - ângulo superior da escápula - ângulo inferior e $\mathrm{D}$ - ângulo superior - ângulo lateral da escápula) que permitiram índice de identificação do sexo em $88,1 \%$.

Palavras-chave: Antropologia Forense. Escápula. DimorfISMO SEXUAL.

Abstract From the paradigm that the determination of post-mortem sex can be performed on any human bone, the present observational analytical study was performed on 195 Brazilian skeleton, of whom 109 were males and 86 were females, existing in the Osteological and Tomographic Biobank Prof. Dr. Eduardo Daruge, of FOP/UNICAMP, which had no growth abnormalities and are in the age range of 18 to 100 years. It aimed to study seven linear measurements in the right scapula: (A - maximum glenoid cavity length, maximum, B - width of the glenoid cavity, C - upper scapula angle - lower angle, D - upper angle - lateral angle of the scapula, E - Lateral angle - lower angle of the scapula, F - more equidistant border of the acromion - upper angle of the scapula and G - length of the acromion) aiming to verify if they are dimorphic and to construct a mathematical model for the determination of post-mortem sex. The results indicated that all measures studied are dimorphic and it was possible to establish a mathematical model with four measures (A - maximum glenoid cavity length, maximum, B - width of the glenoid cavity, C - upper scapula angle - lower angle, D -upper angle - lateral angle of the scapula) which allowed a sex identification index of $88.1 \%$.

Keywords: Forensic Anthropology. Scapula. Sexual dimorPHISM. 


\section{INTRODUÇÃO}

A Antropologia Forense tem a finalidade de promover a identificação do sexo e ancestralidade, além da estimativa da estatura e idade de um indivíduo (KIRAN et al., 2015), ${ }^{1}$ servindo como importante auxílio na identificação post mortem. Com relação ao sexo, pesquisas científicas demonstram que foram encontrados dimorfismo em vários ossos, como no crânio (AMIN e OTHMAN, 2014), ${ }^{2}$ clavícula (MEDIAVILLA, 2016), ${ }^{3}$ patela (ESCORCIA HERNÁNDEZ et al., 2007) $)^{4}$, tíbia (SLAUS et al., 2013) ${ }^{5} \mathrm{e}$ mandíbula (WANKHEDE et al., 2015). ${ }^{6}$

Deve-se destacar que modificações em ossadas, por fatores como a decomposição e processos tafonômicos, evidenciam a necessidade de se realizarem o maior número de avaliações (avaliações qualitativas e quantitativas-estatística descritiva e regressão logística) em todas as outras partes do esqueleto humano, visando a qualidade e fidedignidade do resultado final (TORIMITSU, 2016). ${ }^{7}$

Observa-se na literatura uma lacuna relativamente ao estudo do dimorfismo sexual na escápula. A cintura escapular é composta pela clavícula e escápula. A escápula é um osso chato triangular que se encontra sobre a superfície póstero-superior da caixa torácica e forma a parte posterior da cintura escapular. Apresenta duas faces (anterior e posterior), três bordos (medial, lateral e superior) e três ângulos (superior, inferior e lateral). No seu canto lateral superior, mostra uma depressão em forma de cupê (fossa glenoide), que propicia um encaixe para a cabeça do úmero. A superfície posterior, convexa, é dividida por uma superfície horizontal (espinha escapular), que se estende la- teralmente para formar o acrômio (projeção que sobrepõe a fossa glenoide). $\mathrm{O}$ acrômio se projeta para a frente e se articula com a clavícula, promovendo fixação para os músculos do braço e tórax. A superfície anterior, côncava (formando a fossa subescapular) é medial à fossa glenoide, tendo uma projeção (processo coracoide), que atua como um acessório para músculos e ligamentos (PAPAIONNOU, 2012). ${ }^{8}$

Como todo osso achatado, a escápula é formada quase exclusivamente de tecido compacto e escasso tecido esponjoso (ZHANG, 2016). ${ }^{9}$ Tal situação favorece o encontro da mesma no local de crime, pois os animais decompositores, de médio e grande porte, não encontram grande quantidade de material para a sua alimentação e, dessa forma, a rejeitam.

A fusão completa desse osso ocorre numa fase tardia (23 anos de idade), e o dimorfismo sexual na escápula do ser humano começa a se manifestar na vida fetal, sendo que na vida adulta a escápula feminina permanece com formato grácil e infantil; posteriores alterações morfológicas durante a vida são insignificantes (PAPAIONNOU, 2012). ${ }^{10}$

O presente estudo, a partir do paradigma de que a determinação do sexo pode ser realizada em qualquer osso humano (DABBS e MOORE-JANSEN, 2010), ${ }^{11}$ buscou avaliar o dimorfismo sexual de sete medidas lineares de 195 escápulas direitas, visando verificar se as mesmas são dimórficas e construir um modelo matemático para a determinação do sexo.

\section{MATERIAIS E MÉTODOS}

\section{Amostra}

Esta pesquisa foi submetida e aprovada pelo Comitê de Ética em Pesquisa da Facul- 
dade de Odontologia de Piracicaba - Universidade de Campinas, sob o protocolo $\mathrm{n}^{\circ}$. 138/2014 (CAAE 38522714.6.0000.5418), tendo sido realizada em 195 ossadas, sendo 109 masculinas e 86 femininas (de predominância étnica faioderma e leucoderma), pertencentes ao Biobanco Osteológico e Tomográfico Prof. Dr. Eduardo Daruge, da FOP/UNICAMP), com procedência conhecida e de absoluta certeza quanto à idade, sexo e ancestralidade. As medições foram feitas somente em ossos íntegros.

As medições foram realizadas por três avaliadores.

Para a realização das medidas, utilizou-se paquímetro digital de precisão (marca Stainless - hardened ${ }^{\circledR} 150 \mathrm{~mm}$ Mauá - São Paulo, Brasil), observando-se que a cada medida as pontas do aparelho eram unidas e o calibrador deixado no número zero.
Das ossadas, foram selecionadas apenas escápulas do lado direito, sendo que em cada uma foram efetuadas sete medidas nomeadas de A, B, C, D, E, F e G, sendo:

- A - comprimento máximo da cavidade glenoidal;

- B - largura máxima da cavidade glenoidal;

- C - ângulo superior da escápula ângulo inferior;

- $\quad \mathrm{D}$ - ângulo superior - ângulo lateral da escápula;

- E - ângulo lateral - ângulo inferior da escápula;

- F - borda mais equidistante do acrômio - ângulo superior da escápula;

- $\mathrm{G}$ - comprimento do acrômio.

Figura 1. Medidas A - comprimento máximo da cavidade glenoidal e B - largura máxima da cavidade glenoidal.

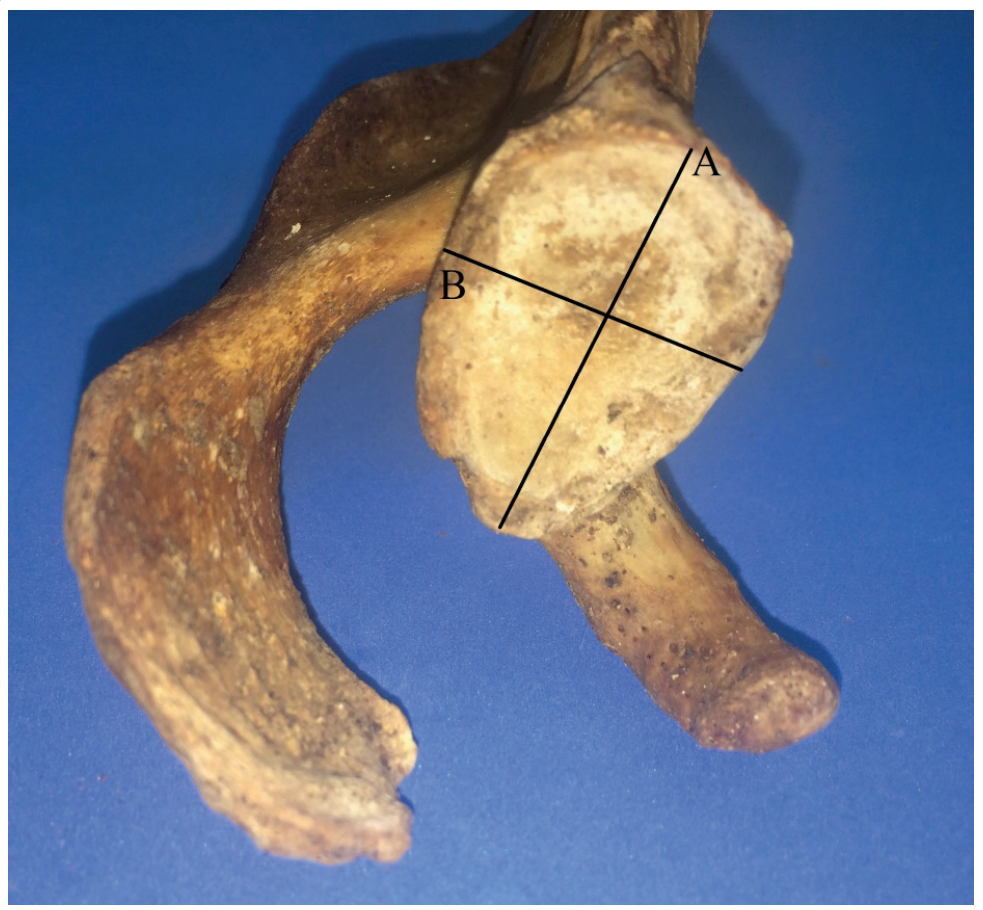


Figura 2. Medidas C - ângulo superior da escápula - ângulo inferior, D - ângulo superior - ângulo lateral da escápula, E - ângulo lateral - ângulo inferior da escápula.

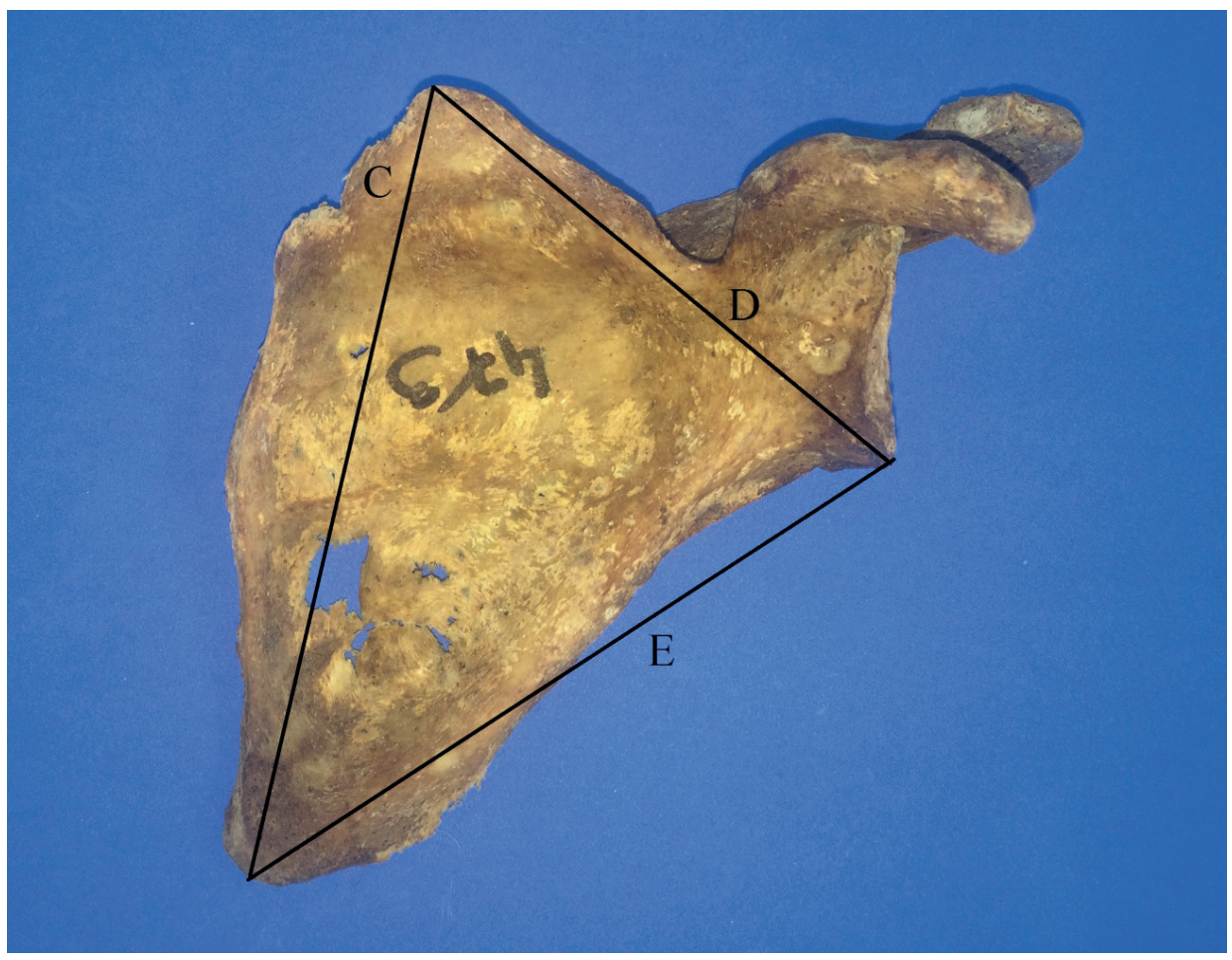

Figura 3. Medidas F - borda mais equidistante do acrômio - ângulo superior da escápula e por último, G - comprimento do acrômio.

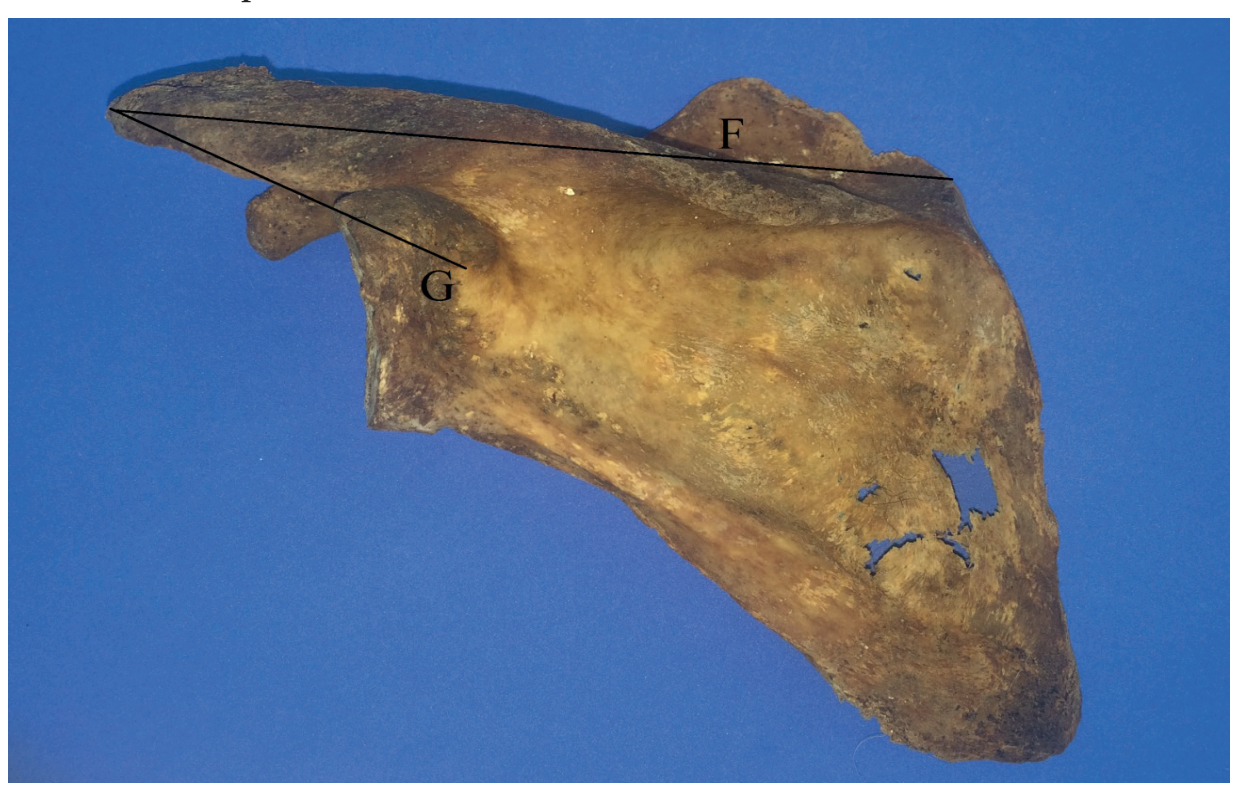




\section{CalibraÇÃo intra E INTEREXAMINADOR}

Antes do início das medições, procedeu-se previamente à calibração inter e intra-examinador, sendo realizada em três períodos distintos em 25 ossadas, com intervalo entre elas de um mês, de forma a se aplicar a análise de correlação intraclasse segundo Szklo e Nieto (2000) para as avaliações inter e intraexaminador.

\section{Análise estatística dos DADOS OBTIDOS}

Foi realizado o teste de Kolmorow-Smirnov e Levene para avaliar a homogeneidade e normalidade; também se utilizou o teste $t$ de Student não pareado (para a análise da relevância das diferenças entre as médias das medidas masculinas e femininas) e o método Stepwise-Wald para a identificação das medidas a serem empregadas para a obtenção de regressão logística. $\mathrm{Na}$ análise estatística, foi usado o software GRAPHPAD PRISM 7.0.

Para a análise dos resultados, está definido para este estudo o nível de significância de 0,01 (1\%), ou seja, quando $\mathrm{p}<0,01$ a diferença é estatisticamente relevante; se $\mathrm{p}>0,01$ a diferença, é estatisticamente irrelevante.

\section{Resultados}

Com relação à calibração intra e interexaminador, as medidas mostraram reprodutibilidade excelente, pois o ICC foi $\geq 0,75$ (Tabela 1).

Tabela 1. Teste de correlação intraclasse (ICC) para replicabilidade intra e interexaminador.

\begin{tabular}{l|l|l}
\hline & ICC & $\mathrm{p}$ \\
\hline A - comprimento máximo da cavidade glenoidal & 0.99 & $<0.0001$ \\
\hline B - largura máxima da cavidade glenoidal & 0.99 & $<0.0001$ \\
\hline C - ângulo superior da escápula - ângulo inferior & 0.99 & $<0.0001$ \\
\hline D - ângulo superior - ângulo lateral da escápula & 0.99 & $<0.0001$ \\
\hline E - ângulo lateral - ângulo inferior da escápula & 0.97 & $<0.0001$ \\
\hline F - borda mais equidistante do acrômio - ângulo superior da escápula & 0.99 & $<0.0001$ \\
\hline G - comprimento do acrômio & 0.99 & $<0.0001$ \\
\hline
\end{tabular}

Feita a calibração, realizaram-se as demais medições.

As medidas foram submetidas aos testes de Kolmogorov-Smirnov e Levene, que mostraram que os dados apresentaram, respectivamente, normalidade (após transformação) e homocedasticidade (variâncias homogêneas).

Posteriormente, aplicou-se o teste $t$ de Student não pareado para a análise da diferença estatisticamente relevante entre as médias das medidas realizadas nas ossadas do sexo masculino e feminino (Tabela 2). 
Tabela 2. Medidas (média \pm desvio) em função do gênero.

\begin{tabular}{l|l|l|l}
\hline & \multicolumn{2}{l|}{ Sexo (média \pm desvio padrão) } & \\
\hline & $\begin{array}{l}\text { Masculino } \\
(\mathrm{n}=109)\end{array}$ & $\begin{array}{l}\text { Feminino } \\
(\mathrm{n}=86)\end{array}$ & $\mathrm{p}$ \\
\hline A - comprimento máximo da cavidade glenoidal & $39.2( \pm 2.4)$ & $34.8( \pm 3.3)$ & $<0.0001$ \\
\hline B - largura máxima da cavidade glenoidal & $28.1( \pm 2)$ & $24.2( \pm 2.7)$ & $<0.0001$ \\
\hline C - ângulo superior da escápula - ângulo inferior & $152.9( \pm 11.9)$ & $131.6( \pm 15)$ & $<0.0001$ \\
\hline D - ângulo superior - ângulo lateral da escápula & $85.5( \pm 12)$ & $74( \pm 10)$ & $<0.0001$ \\
\hline E - ângulo lateral - ângulo inferior da escápula & $134.2( \pm 11.2)$ & $118.4( \pm 12.4)$ & $<0.0001$ \\
\hline F - borda mais equidistante do acrômio - & $134.1( \pm 11.8)$ & $120( \pm 12.6)$ & $<0.0001$ \\
\hline ângulo superior da escápula & $55.6( \pm 4.7)$ & $51.4( \pm 9.2)$ & $<0.0001$ \\
\hline G - comprimento do acrômio & & &
\end{tabular}

A Tabela 2 revela que houve nítido efeito do sexo nas medidas, sendo que todas as medidas foram maiores nos homens, sendo as diferenças consideradas estatisticamente relevantes $(\mathrm{p}<0,01)$.

O terceiro passo foi obter pelo método Stepwise - Wald as medidas a serem utilizadas para a fórmula de regressão logística (Tabela 3).

Tabela 3. Análise de regressão logística para o sexo.

\begin{tabular}{l|l|l|l|l|l}
\hline & Coeficiente & Erro padrão & Wald & Valor de $\mathbf{p}$ & Coeficiente padronizado \\
\hline $\mathrm{A}$ & 0.32 & 0.12 & 7.53 & 0.006 & 1.37 \\
\hline $\mathrm{B}$ & 0.36 & 0.13 & 7.35 & 0.007 & 1.43 \\
\hline $\mathrm{C}$ & 0.077 & 0.025 & 9.75 & 0.002 & 1.08 \\
\hline $\mathrm{D}$ & 0.074 & 0.026 & 8.33 & 0.004 & 1.08 \\
\hline Constante & -37.89 & 5.60 & 45.74 & 0.000 & 0.000 \\
\hline
\end{tabular}

Considerando a probabilidade de acerto ao acaso, os dados revelaram uma porcentagem de $55,1 \%$ de chance de acertar o sexo. A regressão revelou que o modelo composto pelas medidas A, B, C e D foi melhor (Qui-quadrado=147.5, p $<0.0001$ ) para predizer o sexo do que o acaso.

As medidas $\mathrm{E}(\mathrm{p}=0.94), \mathrm{F}(\mathrm{p}=0.14)$ e $\mathrm{G}(\mathrm{p}=0.77)$ não foram importantes para o modelo. $\mathrm{O} \mathrm{R}^{2}$ de Nagelkerke mostrou que as variáveis são responsáveis por $73,5 \%$ da variação encontrada no sexo. Além disso, o teste de Hosmer e Lemeshow revelou que o modelo foi adequado $(\mathrm{p}=0.98)$. 
Assim o modelo matemático obtido é:

$$
\text { Sexo }=-37.89+(0.32 \times A)+(0.36 \times B)+(0.077 \times C)+(0.074 \times D)
$$

Aplica-se então o modelo de regressão logística à amostra e considerando o sexo masculino como " 1 " e o feminino como "0" para efeito do cálculo (sendo que valores maiores que 0,5 seriam considerados como "masculino" e menores como "feminino"), obtendo-se o percentual de predição do sexo (Tabela 4).

Tabela 4. Sexo pela predição obtido com o logito.

\begin{tabular}{l|l|l|l|l}
\hline & & \multicolumn{2}{|l|}{ Predição pela fórmula } & \\
\hline & & Feminino & Masculino & Percentagem correta \\
\hline Sexo real & Feminino & 69 & 14 & 83.1 \\
\hline & Masculino & 8 & 94 & 92.2 \\
\hline & & & Percentagem geral correta & 88.1 \\
\hline
\end{tabular}

A tabela revela que o método resulta em percentual de identificação de $88.1 \%$, mostrando-se, portanto, eficaz na predição do sexo.

\section{DISCUSSÃo}

O primeiro estudo sobre identificação de dimorfismo sexual por meio da escápula data de 1894, e foi realizado por Thomas Dwight que, ao analisar diferenças entre as escápulas masculina e feminina, relatou a utilidade da métrica da escápula na estimativa do sexo, usando o comprimento máximo da escápula e a altura da fossa glenoide. Assim, nas escápulas femininas, o comprimento máximo da escápula teria que ser $<140 \mathrm{~mm}$, enquanto que para as masculinas teria que ser $>170 \mathrm{~mm}$ (DABBS e MOORE-JANSEN, 2010). ${ }^{12}$

Acredita-se que devido à grande miscigenação brasileira (de predominância étnica faioderma e leucoderma) tal estudo seja inválido.
Destaca-se que na população mundial há uma média de 10\% a 20\% (COMA, 1999) ${ }^{13}$ de indivíduos indiferenciados e se supõe que muitos indivíduos se situam na categoria intermediária (de 140 a $170 \mathrm{~mm}$ ). Deduz-se, dessa forma, a necessidade de se validar o mesmo, por meio de novos estudos.

No presente trabalho, conseguiu-se verificar que todas as medidas realizadas são sexualmente dimórficas.

Sabe-se que um dos objetivos dentro da Antropometria Forense é o estudo do dimorfismo sexual, sendo que no Brasil faz-se muito importante que todos os ossos tenham estudos realizados ou validados em coleções recentes e representativas da população miscigenada brasileira. 
O modelo de regressão logística obtido neste trabalho tem grau de acerto de $88,1 \%$, no mesmo sentido de Zhang (2016), ${ }^{14}$ que obteve em sua análise estatística índice de acerto de $86,7 \%$ e uma polarização do sexo de $3,1 \%$. Já os estudos de Dabbs e Moore-Jansen (2010), ${ }^{15}$ na coleção de cadáveres WSU de 32 indivíduos, obteve precisão de $83,4 \%$.

Nos índices de acerto de Papaioannou (2017),${ }^{19}$ no qual a escápula foi explorada por meio de dados geométrico-morfológicos (GM), a precisão obtida foi de $65 \%$.

Porém, os dados observados no presente estudo não são concordes com a coleção de Hamann-Todd, que produziu precisão global de $95,7 \%$ e não são concordes com os estudos de Dabbs e Moore-Jansen (2010), ${ }^{16}$ que, ao avaliarem 80 ossadas da coleção Hamann-Toodd, obtiveram precisão de 92,5\% dos casos, sem viés contra nenhum dos sexos; o mesmo ocorrendo com Torimitsu (2016), ${ }^{17}$ que apresentou uma taxa de $94,5 \%$ em Stepwise DFA, selecionando as medidas LMSH, LMSL e LGBC da escápula esquerda, e 75,7-91,3\% na DFA uni variada com a equação $\mathrm{Y}_{\mathrm{L}}=0,084 \times \mathrm{LMSH}+0,058$ $\mathrm{x}$ LMLS + 0,187 x LGCB-25,383. De forma semelhante, DFA escalonado, usando a escápula direita, selecionando RMSH, RMLS e RGCB, que gerou taxa de 93,1. Giurazza $(2013)^{18}$ obteve com as fórmulas $\mathrm{H}_{\mathrm{m}}=4247$ * $\mathrm{LSL}+93,74$ e $\mathrm{H}_{\mathrm{F}}=4031{ }^{\star} \mathrm{LSL}+92,38$, uma taxa de $98,2 \%$ (erro absoluto de 1,8\%) nos homens e $98,1 \%$ (erro de 1,9) nas mulheres.

Supõe-se que, nos resultados de índices de acerto superiores a $90 \%$, tal fato se deve ao fato da amostra estudada ser composta exclusivamente de ossadas hiperfemininas e hipermasculinas (muito diferenciadas), situação que não é condizente com a realidade da população atual, na qual há inserido de $10 \%$ a $20 \%$ de indivíduos pouco diferenciados (COMA, 1999). ${ }^{20}$

Por fim, o presente estudo proporciona dados para auxiliar a identificação do sexo em esqueletos brasileiros a partir da escápula, dado que a amostra na qual se realizou a pesquisa tem características semelhantes à da população brasileira, pois em ambas encontramos predominância étnica faioderma e leucoderma.

\section{CONSIDERAÇões FINAIS}

Dos resultados obtidos no presente trabalho, pode-se concluir que:

1 - Todas as sete medidas efetuadas na escápula - comprimento máximo da cavidade glenoidal, largura máxima da cavidade glenoidal, ângulo superior da escápula - ângulo inferior, ângulo superior - ângulo lateral da escápula, ângulo lateral - ângulo inferior da escápula, borda mais equidistante do acrômio - ângulo superior da escápula e comprimento do acrômio - apresentam dimorfismo sexual;

2 - Utilizando-se as medidas comprimento máximo da cavidade glenoidal, largura máxima da cavidade glenoidal, ângulo superior da escápula - ângulo inferior e ângulo superior - ângulo lateral da escápula obteve-se fórmula em regressão logística, que atinge índice de identificação do sexo de $88,1 \%$. 


\section{REFERÊNCIAS}

1. Kiran CS, Ramaswamy P., Swathi E., Smitha B., Sudhakar S. Discriminant canine index - a novel approach in sex determination. Ann Stomatol (Roma), 2015, jul. 28, 6 (2): 43-6.

2. Amin, W., Otham, D. Gender Dimorphism of Crania in Young Jordanian Adults: Discriminant Function Analysis Approach for Gender Prediction. J Current Sur. 2014, set., 4 (3), 76-85.

3. Mediavilla, ER. Determining sex with the clavicle in a contemporary Spanish reference collection: A study on 3D images. Forensic Sci Int, 2016, 1 (10): 261-3.

4. Escorcia Hernández L., Lzcano Medina EM, García Alquicira, BG. Determinación de sexo através de funciones discriminantes de la rótula em esqueletos humanos contemporâneos provenientes de Caltimacán, Tasquillo, Hidalgo. Estudios de Antropología Biológica, 2007 (XIII): 135-145

5. Slaus M., Bedić Z., Strinović D., Petrovečki V. Sex determination by discriminant function analysis of the tibia for contemporary Croats. Forensic Sci Int. 2013, mar. 10, 226 (1-3): 302.e1-4.

6. Wankhede KP, Bardale RV, Chaudhari GR, Kamdi NY. Determination of sex by discriminant function analysis of mandibles from a Central Indian population. J Forensic Dent Sci. 2015, jan./abr.,7 (1): 37-43.

7. Torimitsu, S. Sex estimation based on scapula analysis in a Japanese population using multidetector computer tomography. Rev Forensic Sci Int, 262 (2016) 285.e1-285.e5.

8. Papaioannou, VA. Corrigendum to "Sexual dimorphism of the scapula and the clavicle in a contemporary Greek population: Applications in forensic identification" [Forensic Sci. Int. 217 (2012) 231.e1-231.e7]. Rev Forensic Sci Int. 2017, 270: 183.

9. Zhang, K. Estimation of stature and sex from scapular measurements by three-dimensional volume-rendering technique using in Chinese. J. Legal Med. 2016, 21: 58-63.

10. Papaioannou, VA. Corrigendum to "Sexual dimorphism of the scapula and the clavicle in a contemporary Greek population: Applications in forensic identification" [Forensic Sci. Int. 217 (2012) 231.e1-231.e7]. Rev Forensic Sci Int. 2017, 270: 183.

11. Dabbs, GR.; Moore Jansen, P.H. A Method for Estimating Sex Using Metric Analysis of the Scapula. J Forensic Sci, January 2010, 55 (1): 149-52.

12. Dabbs, GR.; Moore Jansen, P. H. A Method for Estimating Sex Using Metric Analysis of the Scapula. J Forensic Sci, January 2010, 55 (1): 149-52.

13. Coma JMR. Antropologia Forense. Madrid: Ministério de Justicia - Centro de Publicaciones, 1991, 178-85; 569-83 e 604-7.

14. Zhang, K. Estimation of stature and sex from scapular measurements by three-dimensional volume-rendering technique using in Chinese. J. Legal Med. 2016, 21: 58-63.

15. Dabbs, GR.; Moore Jansen, P.H. A Method for Estimating Sex Using Metric Analysis of the Scapula. J Forensic Sci, January 2010, 55 (1): 149-52.

16. Dabbs, GR.; Moore Jansen, P. H. A Method for Estimating Sex Using Metric Analysis of the Scapula. J Forensic Sci, January 2010, 55 (1): 149-52.

17. Torimitsu, S. Sex estimation based on scapula analysis in a Japanese population using multidetector computer tomography. Rev Forensic Sci Int, 262 (2016) 285.e1-285.e5.

18. Giurazza, F. Stature estimation from scapular measurements by CT scan evaluation in an Italian population. Legal Med, 2013, 15: 202-8.

19. Papaioannou, VA. Corrigendum to "Sexual dimorphism of the scapula and the clavicle in a contemporary Greek population: Applications in forensic identification" [Forensic Sci. Int. 217 (2012) 231.e1-231.e7]. Rev Forensic Sci Int. 2017, 270: 183. 
20. Coma JMR. Antropologia Forense. Madrid: Ministério de Justicia - Centro de Publicaciones, 1991, 178-85; 569-83 e 604-7.

\section{DAdOS DOS AUTORES}

Maria Julia Assis Vicentim

Graduanda em Odontologia na Faculdade de Odontologia de Piracicaba (Fop/Unicamp). Piracicaba/SP - Brasil. francesq@unicamp.br

\section{ViViane UlbRicht}

Doutoranda em Odontologia Legal e Deontologia pela Faculdade de Odontologia de Piracicaba (Fop/Unicamp). Coordenadora Local dos Cursos de Extensão na FOP-UNICAMP de Perícias Judiciais. Piracicaba/SP - Brasil. viviulbricht@hotmail.com

\section{Cristiane Martins Schmidt}

Doutoranda em Odontologia Legal e Deontologia pela Faculdade de Odontologia de Piracicaba (Fop/Unicamp). Professora de Medicina Legal da Universidade Metodista de Piracicaba (Unimep). Piracicaba/SP - Brasil. cricaschmidt@yahoo.com

\section{Francisco Carlos Groppo}

Doutor em Odontologia pela Universidade Estadual de Campinas (Unicamp). Professor Titular de Farmacologia da Faculdade de Odontologia de Piracicaba (Fop/Unicamp). Piracicaba/SP - Brasil. francesq@unicamp.br

\section{EDUARDo Daruge Junior}

Doutor em Radiologia Odontologica/Área de Odontologia Legal pela Faculdade de Odontologia de Piracicaba (Fop/Unicamp). Professor Livre-docente de Odontologia Legal da Faculdade de Odontologia de Piracicaba (Fop/Unicamp). Piracicaba/SP - Brasil. darugejr@fop.unicamp.br

\section{Sônia Maria de Stefano Piedade}

Doutora em Agronomia pela Universidade de São Paulo. Professora Assistente Doutora da Escola Superior de Agricultura Luiz de Queiroz (ESALQ/USP). Piracicaba/SP - Brasil. francesq@unicamp.br

\section{João Cesar barbieri Bedran de Castro}

Mestre em Direito pela Pontifícia Universidade Católica de São Paulo e em Biologia Buco-Dental pela Faculdade de Odontologia de Piracicaba (Fop/Unicamp). Procurador do Estado de São Paulo. Piracicaba/SP - Brasil. joaobedran@ig.com.br

\section{Carlos Alberto Sassi Etchegoyen}

Doutor em Biologia Buco Dental pela Faculdade de Odontologia de Piracicaba (Fop/Unicamp). Tem experiência em Odontologia Legal com ênfase nos seguintes temas: ética e legislação odontológica, identificação humana e antropologia forense. Piracicaba/SP - Brasil. francesq@unicamp.br

\section{LUIZ Francesquini JUNior}

Doutor em Clínica Odontológica pela Universidade Estadual de Campinas (Unicamp) e Doutor em Radiologia Odontológica pela mesma instituição. Professor Livre-docente de Odontologia Legal da Faculdade de Odontologia de Piracicaba (Fop/Unicamp). Piracicaba/SP - Brasil. francesq@unicamp.br

Submetido em: 15-1-2018

Aceito em: 2-12-2019 